\title{
Influence of tinnitus on pain severity and quality of life in patients with temporomandibular disorders
}

\author{
Patrícia dos Santos CALDERON 1 , Priscila Brenner HILGENBERG ${ }^{2}$, Leylha Maria Nunes ROSSETTI ${ }^{3}$, João Vítor EI \\ Hetti LAURENTI ${ }^{4}$, Paulo César Rodrigues CONTI
}

\author{
1- DDS, MSc, PhD Professor, Department of Dentistry, Federal University of Rio Grande do Norte, Natal, RN, Brazil. \\ 2- DDS, MSc, Graduate student, Department of Prosthodontics, Bauru School of Dentistry, Bauru, SP, Brazil. \\ 3- DDS, MSc, PhD Graduate student, Department of Prosthodontics, Bauru School of Dentistry, Bauru, SP, Brazil. \\ 4- Undergraduate student, Bauru School of Dentistry, Bauru, SP, Brazil. \\ 5- DDS, MSc, PhD Full Professor, Department of Prosthodontics, Bauru School of Dentistry, Bauru, SP, Brazil.
}

Corresponding address: Profa. Dra. Patrícia dos Santos Calderon - Departamento de Odontologia da Universidade Federal do Rio Grande do Norte - Av. Salgado Filho, 1787 - 59056-000 - Lagoa Nova - Natal - RN - Brazil - Phone: +55(84) 3215-4101 - Email: patriciascalderon@yahoo.com.br

Received: June 02, 2010 - Modification: August 26, 2010 - Accepted: October 26, 2010

\section{ABSTRACT}

\begin{abstract}
bjective: The aim of this cross-sectional study was to evaluate the relationship among pain intensity and duration, presence of tinnitus and quality of life in patients with chronic temporomandibular disorders (TMD). Material and Methods: Fifty-nine female patients presenting with chronic TMD were selected from those seeking for treatment at the Bauru School of Dentistry Orofacial Pain Center. Patients were submitted to the Research Diagnostic Criteria anamnesis and physical examination. Visual analog scale was used to evaluate the pain intensity while pain duration was assessed by interview. Oral Health Impact Profile inventory modified for patients with orofacial pain was used to evaluate the patients' quality of life. The presence of tinnitus was assessed by self report. The patients were divided into: with or without self report of tinnitus. The data were analyzed statistically using the Student's t-test and Pearson's Chi-square test, with a level of significance of $5 \%$. Results: The mean age for the sample was 35.25 years, without statistically significant difference between groups. Thirty-two patients (54.24\%) reported the presence of tinnitus. The mean pain intensity by visual analog scale was 77.10 and 73.74 for the groups with and without tinnitus, respectively. The mean pain duration was 76.12 months and 65.11 months for the groups with and without tinnitus, respectively. The mean OHIP score was 11.72 and 11.74 for the groups with and without tinnitus, respectively. There was no statistically significant difference between groups for pain intensity, pain duration and OHIP scores ( $p>0.05)$. Conclusion: Chronic TMD pain seems to play a more significant role in patient's quality of life than the presence of tinnitus.
\end{abstract}

Key words: Tinnitus. Quality of life. Pain. Temporomandibular joint disorders.

\section{INTRODUCTION}

The word tinnitus is of Latin origin, meaning "to tinkle or to ring like a bell". Tinnitus is the perception of sound in the absence of an apparent acoustic stimulus. People describe hearing different sounds: ringing, crickets, whooshing, pulsing, ocean waves, buzzing, and dial tones.

Tinnitus is a common finding. Almost everyone experiences a mild form of tinnitus once in awhile that only lasts a few minutes. However, constant or recurring tinnitus is stressful and can have a profound impact on patients' lives ${ }^{26}$. One third of all adults report experiencing tinnitus at some time in their lives. Ten to $15 \%$ have prolonged tinnitus requiring medical evaluation ${ }^{12}$.

It is not a specific disease entity, but rather a symptom, with many potential causes. Although there are several theories regarding the pathophysiology of tinnitus, the precise mechanism remains to be elucidated ${ }^{\text {. }}$. Tinnitus may be related to otological, neurological and traumatic causes, adverse effects of drugs, nutritional deficiencies, metabolic disturbances, dietary, depression and temporomandibular disorders (TMD) 7 .

Patients with TMD report a higher prevalence 
of tinnitus than do age matched controls ${ }^{8}$. Patients with TMD who suffer from tinnitus seem to have more muscle tenderness to palpation and higher stress levels compared to those without tinnitus ${ }^{3,5}$. Several hypotheses have been proposed for the association between TMD and tinnitus, but no consensus for any single theory has been reached ${ }^{27}$. The first theory was proposed by $\operatorname{Costen}^{10}(1997)$, who believed that the loss of posterior teeth and vertical dimension of occlusion (VDO) could increase the pressure over the ear structures and cause otologic symptoms. Pinto 25 (1962) described a second theory, the existence of a "tiny-ligament", which could be responsible for the otologic symptoms in TMD patients. The third theory was proposed by Myrhaug ${ }^{23}$ (1964), that a muscular TMD could cause a secondary hypertrophy of the tensor tympani and tensor veli palate muscles, generating aural symptoms. Nowadays, the most acceptable is the sensory-motor theory, which suggests that tinnitus modulation can occur by muscular contractions, such as when palpating myofascial trigger-points $3,6,19$.

This cross-sectional study assessed patients with TMD with or without coexisting tinnitus and aimed at evaluating the influence of the presence of tinnitus on the quality of life as well on the intensity and duration of pain of the selected patients with TMD.

\section{MATERIAL AND METHODS}

\section{Sample selection and assessment:}

Fifty-nine patients were selected from individuals seeking treatment for orofacial pain at Bauru School of Dentistry Orofacial Pain Center.

Inclusion criteria were: 1) history of TMD for more than 6 months; 2) pain occurring daily or almost daily for the last month; 3) female gender.

All patients were evaluated initially by the anamnesis and physical examination of the Research Diagnostic Criteria for TMD (RDC/TMD), and were then classified into having muscle and/or joint disorders according to the guidelines of RDC.

The presence of tinnitus and duration of pain were determined by the RDC/TMD questionnaire, which allowed obtaining the self-report of ear noises by the question "Do you have noises or ringing in your ears?" and the self report of pain duration by the question "How many years ago did your facial pain appear for the first time?". The RDC protocol was applied to all patients equally by only one experienced and trained dentist.

The pain intensity was obtained through a visual analog scale (VAS) ranging from 0 to 100 $\mathrm{mm}$. To evaluate the impact of TMD on daily life of the patients the Oral Health Impact Profile (OHIP) modified for orofacial pain patients ${ }^{22}$ was used. In this inventory, the final score varies from 0 to 30, and higher scores indicate higher impact of the TMD in patient quality of life.

After that, the patients were divided into two groups: TMD with complaints of tinnitus or TMD without complaints of tinnitus. All subjects gave informed consent to procedures approved by the Ethics Committee of Bauru School of Dentistry.

\section{Statistical analysis}

The relationship between the presence of tinnitus and the severity of pain (duration and intensity of pain) and the presence of tinnitus and the patients' quality of life were analyzed using the Student's $t$ test. In addition, the association between the presence of tinnitus and the RDC classification was evaluated by Pearson's Chi-square test. The level of significance was set at $5 \%$.

\section{RESULTS}

Fifty-nine patients with ages varying from 17 to 52 years (mean age of 35.25 years) were evaluated. Through the findings of RDC/TMD, the duration of pain was found to vary from 6 months to 32 years (mean duration of 6 years). Self report of tinnitus was observed in $32(54.24 \%)$ patients. The pain intensity for all patients varied from 42 to 100 in the VAS (mean value of 75.56), and the OHIP score varied from 0 to 30 for all sample (mean value of 11.73). Table 1 shows the mean age, pain intensity, pain duration and OHIP scores for the groups.

Statistical analysis showed that there was no statistically significant difference between the groups for age $(p=0.75)$, pain intensity $(p=0.42)$ and duration $(p=0.57)$ and OHIP score $(p=0.99)$.

Table 1- Age (years), intensity (visual analog scale) and duration (months) of pain and quality of life (Oral Health Impact Profile score) by groups

\begin{tabular}{lccc}
\hline & TMD with tinnitus & TMD without tinnitus & P values \\
\hline Age & 34.84 & 35.74 & 0.75 \\
Pain intensity & 77.10 & 73.74 & 0.42 \\
Pain duration & 76.12 & 65.11 & 0.57 \\
Quality of life & 11.72 & 11.74 & 0.99 \\
\hline
\end{tabular}

$\mathrm{TMD}=$ temporomandibular disorders 
Table 2- Research Diagnostic Criteria for temporomandibular disorders (RDC/TMD) classification by groups

\begin{tabular}{lcc}
\hline & TMD with tinnitus & TMD without tinnitus \\
\hline Muscle TMD & 7 & 8 \\
Joint TMD & 3 & 1 \\
Muscle and Joint TMD & 22 & 18 \\
\hline
\end{tabular}

The RDC/TMD classification for this sample shows that 15 patients were classified into having muscle disorder only, 4 patients were classified into having joint disorder only and 40 patients were classified into having muscle and joint disorders.

Table 2 shows the RDC/TMD classification according to the presence or absence of tinnitus. Pearson's Chi-square test showed that there was no statistically significant difference between the groups for the RDC/TMD classification $(p=0.59)$.

\section{DISCUSSION}

According to previous studies, tinnitus can be associated with depression and anxiety ${ }^{4,13}$, sleep disorders ${ }^{11}$ or even lead to suicide ${ }^{16}$. Fortunately, there are not many patients who really suffer from tinnitus, and it is thought that the attention given to the symptom can exacerbate or even perpetuate its existence ${ }^{14}$.

Patients with tinnitus need more than just a diagnosis or physical evaluation. They need advice on their healthcare options, encouragement to seek different treatments, and a commitment from the professional.

Tinnitus can be caused by several reasons, including presbycusis, which generally affects the elderly and is also related to age-related hearing loss $^{1}$. For this reason, it is very common to find elderly patients with tinnitus complaints ${ }^{17}$. However, when a TMD population is evaluated, the mean age of patients with tinnitus is lower because of the TMD distribution in the population. Our findings are in accordance with those of previous investigations ${ }^{7,15}$ as the mean age of patients with tinnitus was about 34 years and was not different from those without tinnitus. This might have occurred because in the present sample only patients with TMD were selected.

In the present study, pain intensity was higher for the TMD and tinnitus group, although it was not statistically significant. A similar result was found by Camparis, et al. ${ }^{7}$ (2005), where patients with tinnitus also had higher pain intensity than the control group. However, it should be held in mind that both tinnitus and TMD have a fluctuating nature and may have different characteristics of intensity throughout time. They both are influenced by psychological conditions and have other brain areas that may be involved in their perception and modulation ${ }^{20}$. Modulation is brain's ability of diminishing pain and/or tinnitus perception by altering the intensity of nociceptive stimuli ${ }^{9}$.

The mean pain duration of tinnitus patients is about 55 to 83 months of pain, according to previous studies ${ }^{6,7}$. In our study the mean pain duration was of 77 months for patients with tinnitus, which was not statistically different from patients without tinnitus. This might have occurred because the sample was comprised of patients diagnosed as having TMD with similar characteristics, and then divided into tinnitus and non-tinnitus groups. Besides, it seems that both tinnitus and chronic pain produce similar alterations in peripheral and central nervous system, suggesting the presence of a unique mechanism. Such central alterations are central sensitization, neuroplasticity and dysfunction of pain suppression system. Both conditions are subjective sensations, with a multifactorial etiology, which can be modulated and change their intensity throughout time. As they have a strong association with emotional factors, it is thought that other brain areas are activated on their perception. The auditory and sensory motor systems have an influence on afferent activity. Moreover, as chronic pain, tinnitus has peripheral an central contributory factors ${ }^{18,19,21}$. The association between tinnitus and chronic pain can be explained by the interaction of sensory-motor systems, characterizing the most acceptable theory of tinnitus in patients with $\mathrm{TMD}^{6,7}$. Although still controversial, it can be also explained by either anatomic connections or some influence of the sympathetic system ${ }^{6}$.

TMD diagnosis between groups was not statistically different. However, approximately $68 \%$ patients with tinnitus had both muscular and articular TMD. This is in accordance with previous findings ${ }^{6,7}$, according to which patients with tinnitus had significantly more muscles and joint disorders than those without tinnitus. Unfortunately, the literature is scarce on precise information about which TMD classification is most prevalent among patients with tinnitus.

The biopsychosocial model of pain includes the interaction of the psychological, social and biological aspects of an individual, explaining why sometimes a depressive patient can persist for years with chronic pain and/or tinnitus. Furthermore, tinnitus and chronic TMD are thought to be somatic syndromes, which may be also influenced by anxiety ${ }^{3}$. Likewise, 
pain levels are also influenced by depression ${ }^{24}$. For these reasons, tinnitus and chronic pain can impair patient's quality of life. In the present study, there was no difference in scores for OHIP between groups $(p=0.99)$, indicating that for this sample, the presence of tinnitus did not compromise patient's quality of life. This result may be explained because in this sample, patients with tinnitus suffered more from their chronic pain than from tinnitus itself. In this case, tinnitus did not play an important role on patient's daily living, as did chronic pain. To the best of our knowledge, the present study is the first one to evaluate the association between the presence of tinnitus in patients with chronic TMD and quality of life. Tinnitus can have a negative correlation with quality of life $^{2}$. However, our findings do not prove that tinnitus impairs quality of life. Further investigations may improve the understanding of this association.

\section{CONCLUSIONS}

The intensity and duration of TMD pain seem to play a more important role in patient's distress than the presence of tinnitus. In other words, patients with chronic TMD did not seem to have their lives impaired by the presence of tinnitus because chronic TMD may be more disabling than the otologic symptom itself. However, this finding could have been influenced by a methodological limitation of the present study, in which patients with tinnitus were gathered by self report from a previously selected chronic pain patient sample. Further studies with a more homogeneous sample evaluated by an Audiologist and an Otolaryngologist as well as submitted to audiological evaluation and validate questionnaires about tinnitus are necessary for better understanding the association between tinnitus and chronic TMD.

\section{ACKNOWLEDGEMENTS}

Authors wish to thank all the participants for the cooperation and CAPES-Brazil for the financial support.

\section{REFERENCES}

1- Ahmad N, Seidman M. Tinnitus in the older adult: epidemiology, pathophysiology and treatment options. Drugs Aging. 2004;21:297-305.

2- Amini R, Haghani $H$, Masoumi M. Quality of life in the Iranian Blind War Survivors in 2007: a cross-sectional study. BMC Int Health Hum Rights. 2010;10:21.

3- Auvenshine RC. Temporomandibular disorders: associated features. Dent Clin North Am. 2007;51:105-27, vi.

4- Belli S, Belli H, Bahcebasi T, Ozcetin A, Alpay E, Ertem U. Assessment of psychopathological aspects and psychiatric comorbidities in patients affected by tinnitus. Eur Arch Otorhinolaryngol. 2008;265:279-85.
5- Bernhardt O, Gesch D, Schwahn C, Bitter K, Mundt T, Mack F, et al. Signs of temporomandibular disorders in tinnitus patients and in a population-based group of volunteers: results of the Study of Health in Pomerania. J Oral Rehabil. 2004;31:311-9.

6- Bezerra Rocha CA, Sanchez TG, Tesseroli de Siqueira JT. Myofascial trigger point: a possible way of modulating tinnitus. Audiol Neurootol. 2008;13:153-60.

7- Camparis CM, Formigoni G, Teixeira MJ, Siqueira JT. Clinical evaluation of tinnitus in patients with sleep bruxism: prevalence and characteristics. J Oral Rehabil. 2005;32:808-14.

8- Chole RA, Parker WS. Tinnitus and vertigo in patients with temporomandibular disorder. Arch Otolaryngol Head Neck Surg. 1992;118:817-21.

9- Conti PCR, Pertes RA, Heir GM, Narsi C, Cohen HV, Araújo CRP. Orofacial pain: basic mechanisms and implication for successful management. J Appl Oral Sci. 2003;11:1-7.

10- Costen JB. A syndrome of ear and sinus symptoms dependent upon disturbed function of the temporomandibular joint. 1934. Ann Otol Rhinol Laryngol. 1997;106:805-19.

11- Folmer RL, Griest SE. Tinnitus and insomnia. Am J Otolaryngol. 2000;21:287-93.

12- Heller AJ. Classification and epidemiology of tinnitus. Otolaryngol Clin North Am. 2003;36:239-48.

13- Holgers KM, Erlandsson SI, Barrenas ML. Predictive factors for the severity of tinnitus. Audiology. 2000;39:284-91.

14- Knobel KA, Sanchez TG. Influence of silence and attention on tinnitus perception. Otolaryngol Head Neck Surg. 2008;138:18-22. 15- Kuttila S, Kuttila M, Le Bell Y, Alanen P, Suonpaa J. Recurrent tinnitus and associated ear symptoms in adults. Int J Audiol. 2005;44:164-70.

16- Lewis JE, Stephens SD, McKenna L. Tinnitus and suicide. Clin Otolaryngol Allied Sci. 1994;19:50-4.

17- Michikawa T, Nishiwaki Y, Kikuchi Y, Saito H, Mizutari K, Okamoto $M$, et al. Prevalence and factors associated with tinnitus: a community-based study of Japanese elders. J Epidemiol. 2010;20:271-6.

18- Møller AR. Neural plasticity in tinnitus. Prog Brain Res. 2006;157:365-72.

19- Møller AR. Pathophysiology of tinnitus. Otolaryngol Clin North Am. 2003;36:249-66, v-vi.

20- Møller AR. Similarities between chronic pain and tinnitus. Am J Otol. 1997; 18:577-85.

21- Møller AR. Similarities between severe tinnitus and chronic pain. J Am Acad Audiol. 2000;11:115-24.

22- Murray $H$, Locker D, Mock D, Tenenbaum HC. Pain and the quality of life in patients referred to a craniofacial pain unit. J Orofac Pain. 1996;10:316-23.

23- Myrhaug $\mathrm{H}$. The incidence of ear symptoms in cases of malocclusion and temporo-mandibular joint disturbances. $\mathrm{Br}$ J Oral Surg. $1964 ; 2: 28-32$.

24- Penna PP, Recupero M, Gil C. Influence of psychopathologies on craniomandibular disorders. Braz Dent J. 2009;20:226-30.

25- Pinto OF. A new structure related to the temporomandibular joint and middle ear. J Prosthet Dent. 1962;12:95-103.

26- Staecker $\mathrm{H}$. Tinnitus evaluation and treatment: assessment of quality of life indicators. Acta Otorhinolaryngol Belg. 2002;56:3556.

27- Wright EF, Bifano SL. Tinnitus improvement through TMD therapy. J Am Dent Assoc. 1997;128:1424-32. 